\title{
Solvent crystallization of palm based dihydroxystearic acid with isopropyl alcohol: effects of solvent quantity and concentration on particle size distribution, crystal habit and morphology, and resultant crystal purity
}

\begin{abstract}
Crude dihydroxystearic acid was prepared from palm based oleic acid and was then solvent purified with isopropyl alcohol in a custom fabricated simultaneous batch crystallizer unit. The crystallized dihydroxystearic acid was a functional ingredient that acted as multipurpose intermediate for synthesis of various fine chemicals, cosmetics and personal care products. The effects of solvent quantity and concentration on particle size distribution, crystal habit and morphology, and resultant crystal purity were studied. The crystals were purer but smaller and the span of the distribution curve was wider at higher solvent quantity and concentration. Through scanning electron microscopy and X-ray diffraction, it was observed that the crystals agglomerated into plate-like (flaky) habit with triclinic crystal structure. Solvent crystallization with $80 \%$ IPA at $20^{\circ} \mathrm{C}$ and solute:solvent ratio of 1.0:1.0 was the most optimized and efficient, producing 6 -DHSA crystals that has high resistance against fat exudation during vacuum filtration process.
\end{abstract}

Keyword: Dihydroxystearic acid; Solvent crystallization; Simultaneous batch crystallizer; Functional ingredient; Process optimization 